\title{
Prospective annual detection rate of high energy gamma ray bursts with LHAASO-WCDA
}

\author{
Bingqiang Qiao ${ }^{a c}$, Mingming Kang ${ }^{b}$, Yuhua $\mathrm{Yao}^{b c}$, Yiqing Guo ${ }^{c}$, Hongbo $\mathrm{Hu}^{c}$ and \\ Zhiguo Yaoc \\ ${ }^{a}$ Purple Mountain Observatory, Chinese Academy of Sciences \\ Nanjing 210008, P.R.China \\ ${ }^{b}$ College of Physics, Sichuan University \\ Chengdu 610064, P.R. China \\ ${ }^{c}$ Institute of High Energy Physics, Chinese Academy of Sciences \\ Beijing 100049,China \\ E-mail: giaobadihep.ac.cn, kangmmdihep.ac.cn, vaoyhdihep.ac.cn, \\ quovgdihep.ac.cn, huhbdihep.ac.cn, vaozgdihep.ac.cn
}

The $\gamma$-ray bursts (GRBs) are the most violent explosion events in the universe ever since the Big Bang and can be served as the high redshift probes for cosmological study. Particularly, the observations on the $\mathrm{GeV}$ emission have had fruitful application, Such examples include the determination of the extra-galactic background light and stringent constraint on the Lorentz Invariance Violation effect. Owing to the advantages in very large effective area, the low threshold energy, the wide field of view and high duty cycle, the LHAASO-WCDA will have potential sensitivity in discovering the GRBs in $100 \mathrm{GeV}$ energy region. In this work, a sample of GRBs has been generated and examined according to the observation of Fermi experiment. We extrapolate the Fermi spectra to high energy by taking into account the absorption due to the pair production process happening between gamma ray and extragalactic background light. With an assumption that ultra high energy component accounts for $10 \%$ of the total luminosity, we find that the LHAASO-WCDA has a GRB detection rate of $\sim 1 / \mathrm{yr}$.

36th International Cosmic Ray Conference -ICRC2019-

July 24th - August 1st, 2019

Madison, WI, U.S.A. 


\section{Introduction}

The GRBs are the most violent explosion events in the universe ever since the Big Bang. While the long GRBs are most likely associated with the massive stellar collapsing, the short GRBs are usually attributed to the merger of two neutron stars, or one neutron star with one black hole. They are also potential sources responsible for the emission of ultra-high energy cosmic rays, highenergy neutrinos, and gravitational waves. The study of GRBs has been prolific over the past decades. Particularly, the observation of the high energy emission above $10 \mathrm{GeV}$ are important in the study of the extra-galactic background light (EBL) and the fundamental physics like Lorentz invariance violation (LIV). GRBs have opened an unique window to connect the field of stellar, interstellar, and intergalactic astronomy as well as cosmology.

With the new generation of space-borne experiment of Ferimi-LAT, the observation of the high energy of GRBs has been stepping into an new era in study the EBL and search for the Lorentz invariance violation. Fermi-LAT has observed more than 20 GRBs which have the highest photon energy being larger than $10 \mathrm{GeV}$. As one example, GRB130427A was able to produce photons up to $94 \mathrm{GeV}$ at the observation ( $126 \mathrm{GeV}$ at source after making redshift correction) [W]. It is unknown up to what energy the spectrum extends, as present-day observations are limited by effective area, in the case of space-based instruments. Ground based detectors are important compensator for the GRB observation when gamma ray energy is above about $30 \mathrm{GeV}$. Two type of techniques are available for this purpose: Air Cherenkov Telescopes (IACTs) and Extended Air Shower arrays (EAS). IACTs have better sensitivity in observing stable point sources for their higher angular resolution and better background rejection power. However IACTs have limited field of view,which makes it possible only for follow up observations and they have to slew very fast to response a real time alert. Even when slew time is not an issue, small field of view requires it an accurate pointing to the GRB direction. Another disadvantage is that IACTs operate only in good weather, at moonless night which makes the duty cycle of IACTs only 10-15\%. Small field of view and low duty cycle make IACTs an inconvenient and inefficient instrument for GRB observation. While EAS arrays have lower sensitivity in observing stable point sources, they have the advantage of very high duty cycle (over 95\%) and very large instantaneous field of view (nominally 2 sr) so they are most suitable for observing GRBs which explode at unexpected time and from unexpected direction. The most powerful EAS arrays in operation is the HAWC experiment using Water Cherenkov technology. However, after taking data for more than 5 years, none GRB has been observed until now. The Large High Altitude Air Shower Observatory (LHAASO), which is currently under construction, is 3.5 time larger and $300 \mathrm{~m}$ higher than HAWC [0]. The effective area is an order of magnitude higher than HAWC at energy of $30 \mathrm{GeV}$ [3], 田]. Moreover, to improve the sensitivity at low threshold energy, a larger PMT with a diameter of $20 \mathrm{inch}$ will be instrumented in the $70 \%$ of LHAASO-WCDA pools.

In this work, we calculate the annual detection rate of GRBs under a conservative assumption that all LHAASO-WCDA is equipped with 8 inches PMTs. The paper is organized in the following way. In Sec. 2, we describe LHAASO experiment in details. Sec. 3 describes the details of GRBs simulation and Sec. 4 describes the significance calculation for each simulated GRB event which involves atmospheric and detector effects. Accordingly, annual detection rate is obtained. Lastly the conclusions are presented in Sec. 5. 


\section{LHAASO Experiment}

LHAASO, locating at DaoCheng, in Sichuan Province of China, is under construction. It is composed of three components: KM2A, WCDA and WFCTA [వ] as shown in Fig.W. Their performances are described as follows.

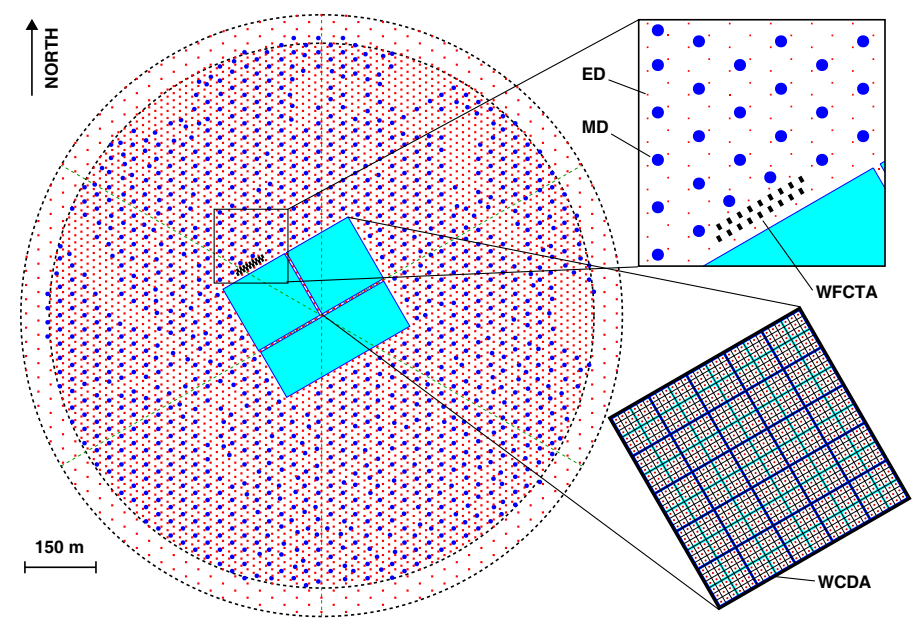

Figure 1: Layout of LHAASO experiment.

\subsection{LHAASO-KM2A}

Covering an area of $1 \mathrm{~km}^{2}$, KM2A is composed of 5195 Scintillator Electron Detectors (EDs) and 1221 Muon Detectors (MDs). The ED array serves to determine the shower direction and energy by recording the arrival time and the density of the secondary electromagnetic particles from air showers. The MD array is used to distinguish between the hadronic and electromagnetic showers via employing their different muon numbers.

\subsection{LHAASO-WCDA}

WCDA is constructed at the center of KM2A and consist of three water ponds with the effective area of 78,000 $\mathrm{m}^{2}$ and water depth of $4.4 \mathrm{~m}$. Each pond is subdivided into $5 \mathrm{~m} \times 5 \mathrm{~m}$ units, partitioned by black plastic curtains to prevent the light from penetrating to the adjacent units. An 8 inches photomultiplier is faced up and anchored to the floor center to collect Cherenkov lights produced by charged particles in the water. Taking $0.5 \mathrm{TeV}$ energy gamma rays as an example, the effective area is as high as $3000 \mathrm{~m}^{2}$, the angular resolution is 0.6 degree and the energy resolution is $95 \%$. The correspondent values are $10000 \mathrm{~m}^{2}, 0.4^{\circ}, 90 \%$ for $1 \mathrm{TeV}$ gamma rays, and $50000 \mathrm{~m}^{2}$, $0.2^{\circ}, 60 \%$ for $10 \mathrm{TeV}$ gamma rays. For a Crab Nebula like source, the most sensitive energy is around $3 \mathrm{TeV}$, one year 5 sigma sensitivity is about $1 \%$ of the Crab Nebula flux.

\subsection{LHAASO-WFCTA}

WFCTA consists of 12 telescopes to measure the cherenkov or fluorescent lights induced by secondary shower particles. It is mainly designed to study the cosmic ray energy spectrum and 
chemical composition by measuring the event based shower energy and $X_{\max }$ parameter. The latter one characterize the shower depth and is sensitive to the nature of primary cosmic rays.

\section{Modeling of GRB parameters and distributions}

A simulation method is adopted to estimate the detection rate of GRBs. Firstly, a set of GRB events is generated according to the distributions of red shift and spectrum of GRBs obtained from past observations. Secondarily, we extrapolate the spectra to multi-TeV energy and subject it to the EBL absorption calculation.

\subsection{The Redshift Distribution}

In literature [ 6 , the space density of GRBs in the comoving reference frame is usually assumed to be a function of redshift $R_{G R B}(z)$. The validation of this assumption is verified after comparing with GRBs observation. After considering the time dilation, the GRBs rate observed today can be described by following formula,

$$
R(z)=\frac{R_{G R B}(z)}{1+z} \cdot \frac{d V(z)}{d z}
$$

where $\mathrm{dV}(\mathrm{z}) / \mathrm{dz}$ is the derivative of the volume element, the factor $(1+z)^{-1}$ reflects the cosmological time dilation, and the $R_{G R B}(z)$ is the the comoving space density of GRBs in the interval $z$ to $z+d z$. According to [目], we adopt the empirical broken power law.

$$
R_{G R B}(z)=\rho_{0} \begin{cases}(1+z)^{n_{1}} & z \leq z_{1} \\ \left(1+z_{1}\right)^{n_{1}-n_{2}}(1+z)^{n_{2}} & z>z_{1}\end{cases}
$$

where $\rho_{0}$ is the local rate with the value $1.3 \mathrm{Gpc}^{-3} \mathrm{yr}^{-1}$ and the empirical parameters $n_{1}, n_{2}, z_{1}$ are fixed to $2.1,-1.4$ and 3.1 respectively.

After the redshift distribution is ready, we are about to determine the intrinsic property of each GRB.

\subsection{Intrinsic parameters of GRB}

The phenomenological GRB generator used in this work is responsible for the generation of the spectral and temporal behaviours for each GRB event. While in the sub-GeV energies, GRBs spectrum can well be described by the so-called Band function, an extra harder component is usually necessary to take care of the higher energy region. Though this extra component actually exhibits a different temporal behaviour, no exact knowledge is available and this work will simply assume it has the same light curve as that for the low energy components. The temporal property is approximately pictured by $T_{90}$, which is the time needed to accumulate from $5 \%$ to $95 \%$ of the observed photon counts.

Overall gamma rays intensity of an GRB is mainly governed by its isotropic peak luminosity $L_{p}$. By assuming that Lp distribution is independent of red shift, $\Phi_{0}\left(L_{p}\right)$ which stand for the fraction of GRBs in an interval between $\log \left(L_{p}\right)$ and $\log \left(L_{p}\right)+d \log \left(L_{p}\right)$ can be parameterized 
from observations. In this work, a form of broken power law distribution is adopted [ $\square,[\mathbb{8}, \mathbf{6}]$,

$$
\Phi_{0}\left(L_{p}\right)=a \begin{cases}\left(L_{p} / L_{p}^{*}\right)^{\gamma_{1}}, & L_{\text {lower }}<L_{p}<L_{p}^{*} \\ \left(L_{p} / L_{p}^{*}\right)^{\gamma_{2}}, & L_{p}^{*}<L_{p}<L_{\text {upper }}\end{cases}
$$

here $a$ is the normalization constant, the other parameters take fixed values as follows, $L_{\text {lower }}=$ $10^{50} \mathrm{erg} / \mathrm{s}, L_{\text {upper }}=10^{54} \mathrm{erg} / \mathrm{s}, L_{p}^{*}=10^{52.5 \pm 0.2} \mathrm{ergs} / \mathrm{s}, \gamma_{1}=-0.17_{-0.2}^{+0.1}$, and $\gamma_{2}=-1.44_{-0.3}^{+0.6}$ as in [ $[8]$.

Empirically, the intrinsic spectrum is well described by the Band function in sub GeV energy [Q],

$$
N(E)=N_{0} \begin{cases}E^{\alpha} \cdot e^{-E(2+\alpha) / E_{p}}, & E \leq E_{c} \\ E^{\beta} \cdot E_{c} \cdot e^{\beta-\alpha}, & E>E_{c}\end{cases}
$$

here the critical energy $E_{c}=\frac{\alpha-\beta}{2+\alpha} E_{p}, N_{0}$ is the normalization constant, $\alpha$ and $\beta$ are the low and high energy photon indices and $E_{p}$ is the peak energy of the spectrum. The $E_{p}$ is constrained by the $E_{p}-L_{p}$ empirical relationship [ए], प]].

Besides the Band spectrum, an extra hard component has been found necessary for a few multi-GeV GRBs discovered by Fermi-LAT [12], [13, 四. This extra component is the dominant contribution to the high energy emission beyond tens of $\mathrm{GeV}$. In our work, an parameter $R_{\text {extra }}$ is introduced to described the relative luminosity from this extra component [14, [15, [16],

$$
R_{\text {ext }}=L_{\text {ext }} / L_{\text {ave }}
$$

here $L_{\text {ext }}$ is the luminosity of the extra component in the $0.1 \mathrm{GeV}$ to $100 \mathrm{GeV}$ energy range, $L_{\text {ave }}$ is the avarage luminosity with the value $0.3 \cdot L_{p}$. Limited by the effective area, statistics of $\mathrm{GeV}$ photons and GeV GRBs so far detected by Fermi-LAT are rare, both parameters, i.e., the spectral index and luminosity, bear rather high uncertainty. As a result $R_{\text {ext }}$ also has large uncertainty. In this work, a value of 0.1 is adopted in the simulation calculation [144]. The spectral index $\left(\beta_{\text {ext }}\right)$ in this energy region is assumed to be -2 to -1.5 [ए7, [0, [3]].

Another intrinsic property of GRB is the temporal information, characterized by $T_{90}$ which is the time window that GRB allocates its $90 \%$ energy release. Following the work [14, [16], $T_{90}$ is simply related to isotropic energy $E_{i s o}$ and average luminosity $L_{a v e}$ by the following formula,

$$
T_{90}=(1+z) \cdot \frac{E_{\text {iso }}}{L_{\text {ave }}}
$$

$E_{i s o}$ can be determined by $L_{p}$ according to the following formula [ए], प]],

$$
\log E_{\text {iso }}=1.1 \cdot \log L_{p}+0.56
$$

At this stage, all parameters needed to generate the in-situ GRB flux, in-situ spectrum annd light curve are available. To obtain the spectrum at observation, one has to consider the EBL absorption effect accumulated in the long journey from its sources to the earth. 


\subsection{EBL attenuation}

The cosmos is not transparent to very high energy $\gamma$-rays. The dominant contribution to the attenuation comes from $\mathrm{e}^{+} \mathrm{e}^{-}$pair production by the target EBL photons, which leads to the softening of the $\gamma$-ray spectrum. The energy-dependent absorption of $\gamma$-ray can be described as $e^{-\tau(E, z)}$, where $\tau(E, z)$ is the optical depth for $\gamma$-ray in energy E. $\tau(E, z)$ can be calculated by integrating the pair production probability with EBL photons coming from all directions and in all energy along the line of sight,

$$
\begin{aligned}
\tau(E, z) & =\int d l \int d \cos (\theta) \frac{1-\cos \theta}{2} \int \frac{d n(\varepsilon, z)}{d \varepsilon} \\
& \times \sigma_{\gamma \gamma}(E, \varepsilon, \cos \theta) d \varepsilon,
\end{aligned}
$$

where $d l$ is the differential path traversed by the $\gamma$-ray and $\sigma_{\gamma \gamma}(E, \varepsilon, \cos \theta)$ is the pair production cross section. Here $d l$ is related to the changing of red shift in an expanding universe by the following way,

$$
d l=\frac{c \cdot d z}{H_{0} \cdot(1+z) \cdot \sqrt{\Omega_{m}(1+z)^{3}+\Omega_{\Lambda}}},
$$

here $H_{0}, \Omega_{m}$ and $\Omega_{\Lambda}$ are the current Hubble constant, the fraction of matter and dark energy. In this work, we adopt the values of 67.8, 0.308 and 0.692 for each of them respectively. According to [ㄷ], $\sigma_{\gamma \gamma}(E, \varepsilon, \cos \theta)$ is calculated as

$$
\begin{aligned}
\sigma(E, \varepsilon, \cos (\theta)) & =\sigma_{T} \cdot \frac{3 m_{e}^{2}}{2 s} \cdot\left[-\frac{p_{e}}{E_{e}}\left(1+\frac{4 m_{e}^{2}}{s}\right)\right. \\
& +\left(1+\frac{4 m_{e}^{2}}{s}\left(1-\frac{2 m_{e}^{2}}{s}\right)\right) \\
& \left.\times\left(\log \frac{\left(E_{e}+p_{e}\right)^{2}}{m_{e}^{2}}\right)\right],
\end{aligned}
$$

here $\sigma_{T}=6.65 \times 10^{-25} \mathrm{~cm}^{2}$ the Thomson cross section, $s=2 E \varepsilon(1-\cos (\theta))(1+z)^{2}$ is the centerof-mass frame energy square, $E_{e}=\sqrt{s} / 2$ and $p_{e}=\sqrt{E_{e}^{2}-m_{e}^{2}}$ are the center-of-mass frame energy and momentum of the electron.

In formula $3.8, n(\varepsilon, z)$ is the photon number density of EBL at redshift $z$ with energy $\varepsilon$. In the calculation, we adopted a distribution from the work [ए]].

\subsection{Consistency check by the Fermi observations}

Firstly, the redshift $z$ and $T_{90}$ distributions are compared between model and observation as shown in Fig. [l. We can see that the model calculations are consistent with the observations within the experimental uncertainties.

The parameters of Band functions, $\alpha, \beta$, fluence and $E_{p k}$, are shown in Fig. Bl, which are generally consistent with the experimental results.

\section{The Results}

Given the fact that GRB model is well validated by observations, a set of GRBs sample can be generated by according to the assumption on these two parameters and can be used to obtain the detection rate of LHAASO-WCDA experiment. 

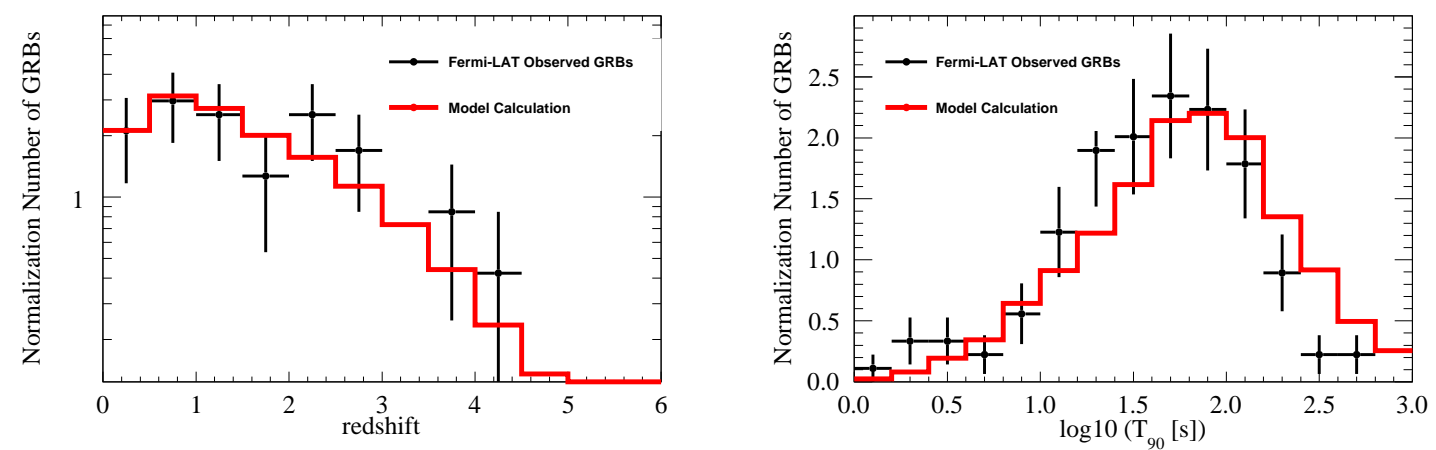

Figure 2: Model calculation of the redshift z (left panel) and $T_{90}$ (right panel) compared with the experimental results.
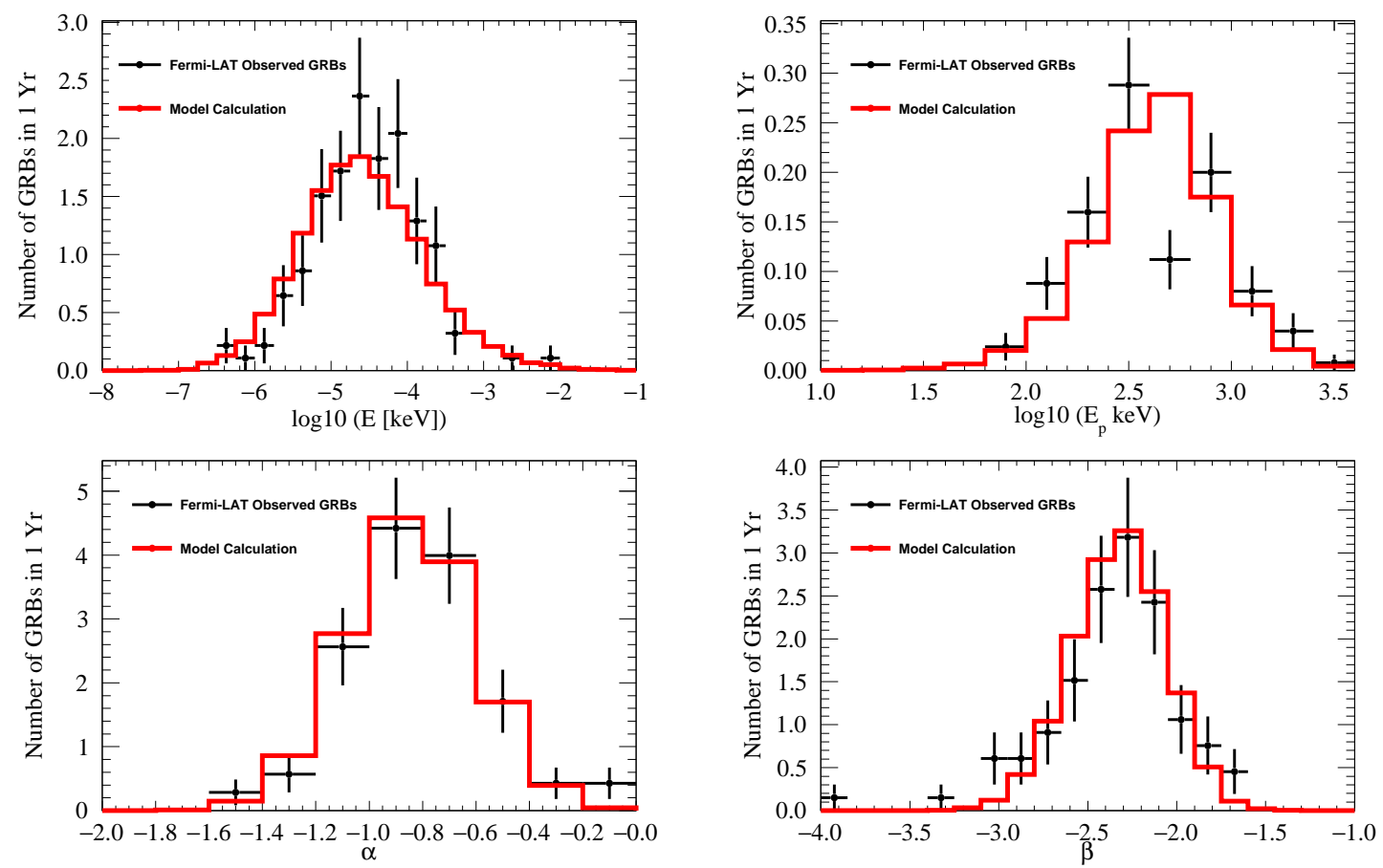

Figure 3: Model calculation of $\alpha$ (left upper panel), $\beta$ (right upper panel), $E_{p}$ and fluence compared with the experimental results.

In the calculation, the signal photons from GRB can be obtained as

$$
N_{S}=T_{90} \int_{E_{\min }}^{E_{\max }} F_{G R B}(E) A_{e f f}(E, \theta) d E
$$

here $T_{90}$ and $F(E)$ has been described in sectoin $3 ; A_{e f f}(E, \theta)$ is the effective area for $\gamma$-rays at the zenith angle $\theta$ and energy E adopted from the work [ [ $⿴ 囗 十$ ]. The zenith angle $\theta$ of the burst are uniformly distributed over the sky and can be randomly generated. The backgrounds $N_{B}$, coming from the CRs, can be calculated similarly as $N_{S}$ by replace the burst flux $F_{G R B}(E)$ with background flux $F_{C R s}(E, \theta)$ and replace the effective area by that of cosmic rays. The significane can be calculated 
based on the signal number of $\gamma$-rays $N_{S}$ and background number of CRs $N_{B}$. A $5 \sigma$ is required to claim the discovery of a GRB by LHAASO-WCDA.

As a result, Fig. 团 shows the model calculation of the detection rate for LHAASO-WCDA. The left plot is the differential detection number of GRBs as a function of the number of detected $\gamma$-rays. The integral detection rate as a function of the detected $\gamma$-rays number is plotted in the right panel. The black, green, blue and red lines are calculated with Band spectrum, Band spectrum for $\beta<-2$, $R_{e x t}=0.1$ and $\beta_{\text {ext }}=-2,-1.5$ respectively. It is obvious that the detection rate of LHAASO-WCDA is only 0.38 and 0.31 for Band spectrum and Band spectrum with $\beta<-2$. When we adopt the extra component with $R_{e x t}=0.1 \beta=-2$ and $\beta=-1.5$, the detection rate of LHAASO-WCDA is improved to be 0.84 and 2.63 per year. Therefore, the detection rate of LHAASO-WCDA should be around 1 per year.
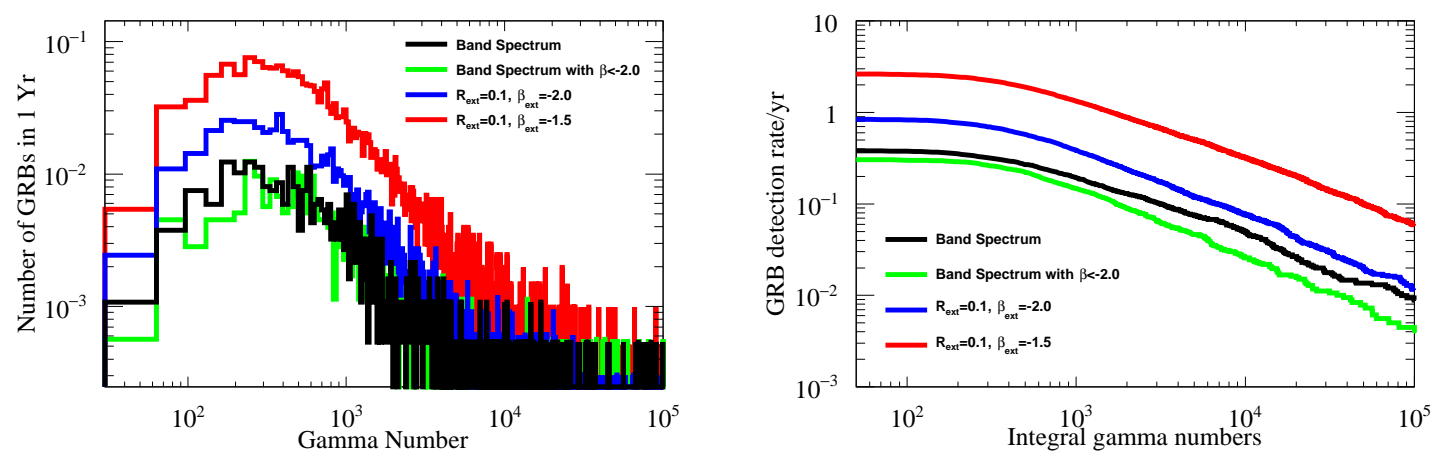

Figure 4: The model calculation of the differential (left panel) and integral (right panel) detection rate for LHAASO-WCDA experiment.

\section{Conclusion}

The high energy emissions of GRBs play important roles in understanding the remaining GRB mysterious, such as the mechanism of their central engine, the ultrahigh lorentz factor $\Gamma$ and the origin of ultra-high-energy CRs. They also have pratical application including the EBL measurement and the test of LIV. In view of this, the observation of the high energy emissions from GRBs becomes great importance. In this work, we perform a simulation calculation to estimate the annual detection rate of GRBs for LHAASO-WCDA experiment. In the simulation, the generated sample of GRBs is firstly examined by Fermi resutls. By doing so, we think that an adequate sample is ready to estimate the detection rate of LHAASO-WCDA experiment. As a result, an encouraging value, i.e., $\sim 1$ GRB per year, is obtained for LHAASO-WCDA under the assumption that extra high energy component accounts $10 \%$ of that of total luminosity.

\section{Acknowledgements}

This work is supported by the Ministry of Science and Technology of China, Natural Sciences Foundation of China $(11705121,11761141001)$ and the National Key Research and Development Program of China(2018YFA0404202). 


\section{References}

[1] M. Ackermann, M. Ajello, K. Asano, et al. Fermi-LAT Observations of the Gamma-Ray Burst GRB 130427A. Science, 343:42-47, January 2014.

[2] Z. Cao. A future project at tibet: the large high altitude air shower observatory (LHAASO). Chinese Physics C, 34:249-252, February 2010.

[3] Joshua Wood. An All-Sky Search for Bursts of Very High Energy Gamma Rays with HAWC. arXiv e-prints, page arXiv:1801.01550, Jan 2018.

[4] X. Bai, B. Y. Bi, X. J. Bi, et al. The Large High Altitude Air Shower Observatory (LHAASO) Science White Paper. arXiv e-prints, page arXiv:1905.02773, May 2019.

[5] Y. Liu, Z. Cao, S. Chen, et al. Expectation on Observation of Supernova Remnants with the LHAASO Project. ApJ, 826:63, July 2016.

[6] David Wanderman and Tsvi Piran. The luminosity function and the rate of Swift's gamma-ray bursts. MNRAS, 406(3):1944-1958, Aug 2010.

[7] M. Schmidt. Luminosities and Space Densities of Gamma-Ray Bursts. ApJ, 523:L117-L120, October 1999.

[8] S. Casanova, B. L. Dingus, and B. Zhang. Contribution of GRB Emission to the GeV Extragalactic Diffuse Gamma-Ray Flux. ApJ, 656:306-312, February 2007.

[9] D. Band, J. Matteson, L. Ford, et al. BATSE observations of gamma-ray burst spectra. I - Spectral diversity. ApJ, 413:281-292, August 1993.

[10] G. Ghirlanda, L. Nava, G. Ghisellini, A. Celotti, and C. Firmani. Short versus long gamma-ray bursts: spectra, energetics, and luminosities. A\&A, 496:585-595, March 2009.

[11] S. Qi and T. Lu. Toward Tight Gamma-Ray Burst Luminosity Relations. ApJ, 749:99, April 2012.

[12] A. A. Abdo, M. Ackermann, M. Ajello, et al. Fermi Observations of GRB 090902B: A Distinct Spectral Component in the Prompt and Delayed Emission. ApJ, 706:L138-L144, November 2009.

[13] M. Ackermann, K. Asano, W. B. Atwood, et al. Fermi Observations of GRB 090510: A Short-Hard Gamma-ray Burst with an Additional, Hard Power-law Component from $10 \mathrm{keV}$ TO GeV Energies. ApJ, 716:1178-1190, June 2010.

[14] G. Ghisellini, G. Ghirlanda, L. Nava, and A. Celotti. GeV emission from gamma-ray bursts: a radiative fireball? MNRAS, 403:926-937, April 2010.

[15] Aurelien Bouvier, Rudy Gilmore, Valerie Connaughton, et al. Prospects of GRB observations for CTA from a phenomenological model. arXiv e-prints, page arXiv:1109.5680, Sep 2011.

[16] Jun Kakuwa, Kohta Murase, Kenji Toma, et al. Prospects for detecting gamma-ray bursts at very high energies with the Cherenkov Telescope Array. MNRAS, 425(1):514-526, Sep 2012.

[17] B. L. Dingus. EGRET Observations of $>30 \mathrm{MeV}$ Emission from the Brightest Bursts Detected by BATSE. Ap\&SS, 231:187-190, September 1995.

[18] R. J. Gould and G. P. Schréder. Pair Production in Photon-Photon Collisions. Physical Review, 155:1404-1407, March 1967.

[19] R. Gilmore. Extragalactic Background Light and Gamma-ray Opacity. In American Astronomical Society Meeting Abstracts \#217, volume 43 of Bulletin of the American Astronomical Society, page 231.02, January 2011. 